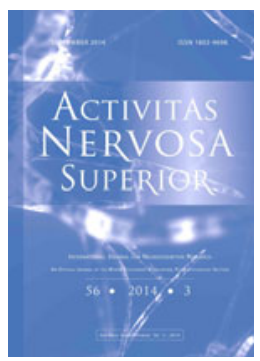

\title{
Telomeres IN THE BRAIN CORTEX OF PATIENTS WITH MAJOR DEPRESSIVE DISORDER
}

\author{
Jean-Raymond Teyssier, ${ }^{*}{ }^{*}, 2$ Sylviane Ragot, ${ }^{1}$ Anne Donzel, ${ }^{1}$ \\ Jean-Christophe Chauvet-Gelinier ${ }^{2}$ \\ Department of Genetics ${ }^{1}$ and Psychiatry Unit, ${ }^{2}$ \\ University Hospital and Faculty of Medicine, \\ University of Burgundy, Dijon, France
}

\begin{abstract}
Telomeres are complex structures formed by the end of the DNA molecule at the tip of each chromosomal arm. The repeated (TTAGGG) telomeric sequence progressively shortens during lifespan because it cannot be replicated as somatic cells divide, and is highly susceptible to breakage by free radicals. Critically shortened telomeres activate the genetic program of cell senescence and/or apoptosis. The telomere length measured in peripheral blood leucocytes is considered a reliable marker of biological age, mortality risk and exposure to various pathological conditions, including cardiovascular disease, dementia, and metabolic syndrome. Telomere erosion has been observed in psychiatric disorders including schizophrenia and mood disorders, suggesting an accelerated aging of 10 to 20 years. Whether this peripheral dynamic is reflected by a similar pattern in the brain remains unknown. To address this issue we have measured the telomere length in the occipital DNA cortex of 24 patients with major depressive disorder and 12 controls (donated by the Stanley Research Institute) by a real time quantitative PCR technique. The mean telomere lengths were identical in the depressed and control groups. Thus, although there is consistent evidence for the role of systemic inflammation and oxidative stress in depression, it must be concluded that the cerebral status of telomeres is not affected. This observation raises the issue of the relation between the psychiatric pathological process, and peripheral and central biomarkers.
\end{abstract}

Key words: Depression; Telomere; Cortex

\section{INTRODUCTION}

Telomeres are complex nucleoprotein structures, capping the linear DNA end at the tip of each chromosome arm (Aubert \& Landsdorp, 2008). They are formed by a loop of GC-rich DNA repeats. In vertebrates, the telomere is composed of a long tract of the repeated TTAGGG sequence, which in humans extends for about 5 to $15 \mathrm{~kb}$. This paper clip like loopends with a short single-stranded DNA overhang, which is highly susceptible to breakage by reactive oxygen species (ROS). The telomere architecture is dynamically stabilized by about ten proteins, which interact in a complex and still unresolved way, with the signaling molecular networks involved in the control of DNA repair, cell proliferation, cell death, and

"Correspondence to: Jean-Raymond Teyssier,e-mail: teyssier@u-bourgogne.fr

[Translated with permission from L'Encephale, 2010, 36, 491-494]

Received September 21, 2012; accepted November 14, 2012; Act Nerv Super 56(3), 89-94; ISSN-1802-9698 
cell senescence. Because of kinetics constraint, the DNA- $\delta$ polymerase which synthesizes the bulk of the DNA is unable to replicate the terminal telomere sequence. The consequence is that telomeres regularly shorten during each cell cycle of somatic cells. Only the telomerase enzyme can autonomously elongate the terminal repeats. The gene encoding this enzyme is expressed during embryogenesis, but is down regulated after birth in virtually all cell types except for stem cells, germ cells, and activated lymphocytes, in which telomerase can only partly prevent telomere shortening. Furthermore, telomeric DNA is especially sensitive to ROS-induced single-strand breaks and oxidation of the deoxyGuanine base to 8oxodeoxyGuanine (8-oxodG), which accelerate telomere erosion in a cumulative way over the lifespan (Houben et al, 2008). There is evidence that oxidative stress associated with chronic inflammatory disease and low-grade systemic inflammation, plays a major role in this ROSmediated telomere shortening ( $\mathrm{O}^{\prime}$ Donovan et al, 2011).The reduction of telomere sequences to a critical length activates interlinked cellular safeguard systems, at crossroads of genome stability surveillance, cell cycle progression, cell death, and tissue renewal, leading to activation of the cell senescence program (Sahin \& DePinho, 2010). Telomeres which monitor both the proliferative history of the cells, and the chronic oxidative stress load, are indicators of the "biological age".

In humans, numerous population studies have measured the mean telomere length (MTL) in the peripheral blood leucocytes. There is a large inter-individual variability in MTL values and attrition rates. Telomeres are longer in women, and MTL is under genetic control (heritability estimate of $60 \%$ ). The MTL measured in different tissues is linearly and inversely correlated with the chronological age, with an average annual erosion rate of roughly 50 base pairs. The values obtained in blood leucocytes varied from more than $10 \mathrm{~kb}$ at birth to about 4 $\mathrm{kb}$ at 80 years. Thus the MTL recorded at a given age reflects the combined effects of individual-specific (genetic) factors, environmental influences, and stochastic aging processes. Results of epidemiological studies have provided evidence that accelerated shortening of telomeres is a reliable risk marker for aging-related pathologies (including cardiovascular disease, hypertension, diabetes, metabolic syndrome, osteoporosis, dementia, and cancer), and is associated with poor clinical outcome (Zhu et al, 2011). More importantly, MTL is not only a disease marker, but also an independent mortality predictor and a mediator of lifespan. As a group, subjects in their sixties-seventies with shortest telomeres for their chronological ages have three times greater risk of death during a follow-up period of about ten years, than those with the longest telomeres (Bakaysa et al, 2007; Fitzpatrick et al, 2011). More recently, the only available live long study using iterative measures at various ages (carried out in finches), has clearly demonstrated that telomere length in early life is a strong predictor of longevity (Heidinger et al, 2012).

In MDD, leucocyte MTL has been reported to be inversely correlated with the duration of the depressive disease, and with the level of oxidative stress and inflammation markers (Wolkowitz et al, 2011). Together with the well-recognized medical comorbidities (metabolic and cardiovascular) observed in MDD (Moussavi et al, 2007), these findings have led to the hypothesis that depression should be recognized as a somatic disease in its own right, which causes premature aging (Wolkowitz et al, 2011). In this context, it has been suggested that the shortening of telomeres in neurons of the cerebral cortex by cumulative oxidative stress, might contribute to the physiopathology of MDD and influence brain aging. To address this issue we have measured the MTL in the occipital DNA cortex of 24 patients with MDD and of 12 controls.

\section{METHOD}

The DNA samples extracted from the occipital cortex (gray matter) of the 36 subjects have been kindly donated by the Stanley Medical Research Institute (Doctors M.B. Knable, E.F. Torrey, M.J. Webster). The population was composed of 24 patients with MDD (12 with 
psychotic characteristics) and 12 controls with no psychiatric disease. The mean ages of the MDD and control subjects were 42.1 and 46.8 years respectively. The mean duration of the disorder was 13 years. Among depressive patients 17 died by suicide. The subjects were matched for sex, age, ethnicity and mean brain $\mathrm{pH}$. All the patients have received antidepressant treatment. The detailed demographic, clinical, and brain tissue characteristics are available at http://www.stanleyresearch.org/brain/. The MTL has been measured by the real time quantitative PCR technique, exactly as previously described (Cawthon, 2002), using a sequence of the $36 \mathrm{~B} 4$ gene as reference. The PCR cycle parameters were: $94^{\circ}$ for 15 seconds, $54^{\circ}$ for 1 minute, and $72^{\circ}$ for 30 seconds ( 35 cycles). The arbitrary unit used for the quantification of the PCR products labeled by a fluorochrome, was the number of PCR cycles producing a fluorescent signal above the detection threshold $(\mathrm{Ct})$.

The results were expressed by the ratio of the mean (triplicates) $\mathrm{Ct}$ values ( $\mathrm{Ct}$ telomere $/ \mathrm{Ct}^{36 \mathrm{~B} 4}$ ) and compared by the Student's t-test. The PCR efficiency and reproducibility have been established by the standard curve method.

\section{RESULTS}

The $\mathrm{Ct}$ ratios of telomeres varied from 0.78 to 0.80 and their means were strictly identical in all tested groups: $0.79( \pm 0.001)$, indicating for the first time that there was no telomere erosion in the cortex of patients with depressive disorder, even in case of long lasting recurring symptoms, psychotic characteristics or suicidal behavior (see Table 1, p. 90).

\section{DISCUSSION}

Four studies including 251 depressed individuals have reported that there was a significant shortening of MTL in the leucocytes of patients with long (tens of years) lifetime MDD duration, equivalent to approximately 7 to 10 years of accelerated aging (Wolkowitz et al, 2011; Simon et al, 2006; Hartmann et al, 2010; Wikgren et al, 2012). No correlation was generally found between MTL and severity of the current MDD episode evaluated by symptoms scale ratings. In one of these studies, MTL was inversely correlated with the blood oxidative stress level and IL-6 concentration (Wolkowitz et al, 2011), and in other one with a hypocortisolemic state (Wikgren et al, 2012). Similarly, lifetime numbers of depressive episodes were significantly correlated with telomere shortening in patients with bipolar II disorder (Elvsashagen et al, 2001). There is also rapid telomere erosion in patients with schizophrenia after 20 years of illness, indicating a premature biological aging of about 25 years (Kao et al, 2008).

Two non-exclusive phenomena may account of our negative results in the brain tissue. Only minor or no shortening of MTL with age has been found in normal gray matter or cerebellum (Nakamura et al, 2007). In Alzheimer's disease, blood telomere attrition was not associated with a similar change in the cerebellum (Lukens et al, 2009). This is intriguing since brain cells are subjected to high levels of oxidative stress. Experimental results have provided evidence that this pattern might depend on a specific adaptative mechanism linking telomere maintenance and DNA repair pathways, which protects neurons against oxidative, metabolic, and excitotoxic insult. The telomere repeat-binding factor 2 (TRF2), a component of the telomere complex, could play a key role in this stabilizing mechanism (Cheng et al, 2007).

On the other hand, whether the chronic peripheral pro-oxidative state consistently identified in MDD is a surrogate of cerebral pathological changes is an unproven assumption. Recently, we have directly addressed this issue by quantifying the expression of eight genes encoding the major oxidative stress response proteins, including detoxifying and repair enzymes, in the dorsolateral prefrontal cortex of the patients whose MTL has been measured in the present study (Teyssier et al, 2011). We found no change in the expression level of these 
genes. In relation to the significance of oxidative stress and brain aging in MDD, one of these genes is worth considering. The OGG1 gene encodes the 8-oxoguanine DNA glycosylase I, which repairs oxidized DNA by eliminating 8-doxoG accumulated under the effects of ROS.

Table 1. Telomere length expressed by the $\mathrm{Ct}^{\text {tel }} / \mathrm{Ct}^{36 \mathrm{~B} 4}$ ratio.

\begin{tabular}{|c|c|c|c|}
\hline Diagnosis & Ct tel & Ct 36b4 & $\mathrm{Ct}^{\mathrm{tel}} / \mathrm{Ct}^{36 \mathrm{~B} 4}$ \\
\hline \multirow{24}{*}{ Depression } & $20.29 *$ & 25.57 & 0.79 \\
\hline & $19.32 *$ & 24.38 & 0.79 \\
\hline & 19.24 & 24.84 & 0.77 \\
\hline & $20.01 *$ & 25.21 & 0.79 \\
\hline & $19.35^{*}$ & 24.83 & 0.78 \\
\hline & $18.91^{*}$ & 24.33 & 0.78 \\
\hline & 19.29 & 24.44 & 0.79 \\
\hline & $19.45^{*}$ & 23.7 & 0.82 \\
\hline & $19.41^{*}$ & 24.64 & 0.79 \\
\hline & $19.49 *$ & 24.54 & 0.79 \\
\hline & 18.6 & 23.43 & 0.79 \\
\hline & 18.85 & 24.03 & 0.78 \\
\hline & 18.95 & 23.89 & 0.79 \\
\hline & 18.7 & 24.1 & 0.78 \\
\hline & 18.55 & 23.6 & 0.79 \\
\hline & 19.01 & 23.95 & 0.79 \\
\hline & 19.37 & 24.57 & 0.79 \\
\hline & $19.61^{*}$ & 24.92 & 0.79 \\
\hline & $18.6^{*}$ & 23.86 & 0.78 \\
\hline & $19.17 *$ & 24.31 & 0.79 \\
\hline & 18.73 & 23.34 & 0.80 \\
\hline & 19.04 & 24.26 & 0.78 \\
\hline & 19.68 & 25.1 & 0.78 \\
\hline & 18.78 & 23.88 & 0.79 \\
\hline \multirow{12}{*}{ Controls } & 20.39 & 25.54 & 0.80 \\
\hline & 19.17 & 24.33 & 0.79 \\
\hline & 19.09 & 24.07 & 0.79 \\
\hline & 18.6 & 23.52 & 0.79 \\
\hline & 18.81 & 24.24 & 0.78 \\
\hline & 19.76 & 24.82 & 0.80 \\
\hline & 19.01 & 24.18 & 0.79 \\
\hline & 19.79 & 24.33 & 0.81 \\
\hline & 19.38 & 24.44 & 0.79 \\
\hline & 19.6 & 24.91 & 0.79 \\
\hline & 19.37 & 24.99 & 0.78 \\
\hline & 19.24 & 24.2 & 0.80 \\
\hline
\end{tabular}

*Depression with psychotic characteristics.

Expression level of this gene is a validated biomarker for in vivo detection of chronic oxidative stress (Loft et al, 2008). Furthermore overexpression of OGG1 is a hallmark of the oxidative stress response activated in the aging human brain (Lu et al, 2004). A high serum 
level of 8-doxoG is associated with severe and recurrent depression (Forlenza et al, 2006), however, according to our results that this peripheral oxidative has a significant repercussion on the brain appears unlikely.

A current model assumes that brain chronic oxidative stress contributes to the pathophysiology of MDD, and is the common mediator of various interacting mechanisms including psychological stress, hypercortisolemia, dopamine metabolism, inflammation, mitochondrial dysfunction, and apoptotic neural loss (Berk et al, 2008). The present findings do not support the hypothesis that the cortex of depressive patients is affected by a pathological oxidative stress, even under severe conditions such as suicide execution or progressive psychosis. Consequently it has become unacceptable to infer the role of oxidative stress in the pathogenesis of mental diseases from the alterations of peripheral blood markers. How the biopsychological stress associated with psychiatric diseases is transduced to the leucocytes of the immune and inflammatory cell compartment (and potentially to other cell types including stem cells) remains to be elucidated.

\section{REFERENCES}

Aubert, G., \& Landsdorp, P.M. (2008). Telomeres and Aging. Physiological Review, 88, 557-579.

Bakaysa, S.L., Mucci, L.A., Slagboom, P.E., Boomsma, D.I., McClearn, G.E., Johansson, B., Pedersen, N.L. (2007). Telomere length predicts survival independent of genetic influences. Aging Cell, 6, 769-774.

Berk, M., Ng, F., Dean, O., Dodd, S., \& Bush, A.I. (2008). Glutathione: a novel treatment target in psychiatry. Trends in Pharmacological Sciences, 29, 346-351.

Cawthon, R.M. (2002). Telomere measurement by quantitative PCR. Nucleic Acid Res, 30, e47.

Cheng, A., Shin-ya, K., Wan, R., Tang, S.C., Miura, T., Tang, H., Khatri, R., Gleichman, M., Ouyang, X., Liu, D., Park. H.R., Chiang, J.Y., \& Mattson, M.P. (2007). Telomere protection mechanisms change during neurogenesis and neuronal maturation: newly generated neurons are hypersensitive to telomere and DNA damage. Journal of Neuroscience, 27, 3722-3733.

Elvsåshagen, T., Vera, E., Bøen, E., Bratlie, J., Andreassen, O.A., Josefsen, D., Malt, U.F., Blasco, M.A., \& Boye, B. (2011). The load of short telomeres is increased and associated with lifetime number of depressive episodes in bipolar II disorder. Journal of Affective Disorders, 135, 43-50. doi: 10.1016/j.ad.2011.08.006.

Fitzpatrick, A.L., Kronmal, R.A., Kimura, M., Gardner, J.P., Psaty, B.M., Jenny, N.S., Tracy, R.P., Hardikar, S., \& Aviv, A. (2001). Leucocyte telomere length and mortality in the Cardiovascular Health Sudy. Journals of Gerontology, 66A, 421-429.

Forlenza, M.J., \& Miller, G.E. (2006). Increased serum levels of 8-hydroxy-2'-deoxyguanosine in clinical depression. Psychosomatic Medicine, 68, 1-7.

Hartmann, N., Boehner, M., Groenen, F., \& Kalb, R. (2010). Telomere length of patients with major depression is shortened but independent from therapy and severity of the disease. Depression and Anxiety, 27, 1111-1116.

Heidinger, B., Blount, J., Boner, W., Griffiths, K., Metcalfe, N., \& Monaghan, P. (2012). Telomere length in early life predicts lifespan. Proceedings of the National Academy of Sciences of the United States of America, 109, 1743-1748.

Kao, H.T., Cawthon, R.M., Delisi, L.E., Bertisch, H.C., Ji, F., Gordon, D., Li, P., Benedict, M.M., Greenberg, W.M., \& Porton, B. (2008). Rapid telomere erosion in schizophrenia. Molecular Psychiatry, 13, 118-119.

Loft, S., Hogh Danielsen, P., Mikkelsen, L., Risom, L., Forchhammer, L., \& Moller, P. (2008). Biomarkers of oxidative damage to DNA and repair. Biochemical Society Transactions, 36, 1071-1076.

Lu, T., Pan, Y., Kao, S.Y., Li, C., Kohane, I., Chan, J., Yankner, B.A. (2004). Gene regulation and DNA damage in ageing human brain. Nature, 429, 883-891.

Lukens, J.N., Van Deerlin, V., Clark, C.M., Xie, S.X., Johnson, F.B. (2009). Comparisons of telomere lengths in peripheral blood and cerebellum in Alzheimer's disease. Alzheimer's and Dementia, 5, 463469.

Moussavi, S., Chatterji, S., Verdes, E., Tandon, A., Patel, V., \& Ustun, B. (2007). Depression, chronic diseases, and decrements in health : results from the World Health Survey. Lancet, 370, 851-858.

Nakamura, K., Takubo, K., Izumiyama-Shimomura, N., Sawabe, M., Arai, T., Kishimoto, H., Fujiwara, M., Kato, M., Oshimura, M., Ishii, A., Ishikawa, N. (2007). Telomeric DNA length in cerebral gray and 
white matter is associated with longevity in individuals aged 70 years or older. Experimental Gerontology, 42, 944-950.

O'Donovan, A., Pantell, M.S., Puterman, E., Dhabhar, F.S., Blackburn, E.H., Yaffe, K., Cawthon, R.M., Opresko, P.L., Hsueh, W.C., Satterfield, S., Newman, A.B., Ayonayon, H.N., Rubin, S.M., Harris, T.B., Epel, E.S. (2011). Cumulative inflammatory load is associated with short leucocyte telomere length in the health, aging, and body composition study. PloS ONE, 6, e19687.

Sahin, E., \& DePinho, R.A. (2010). Linking functional decline of telomeres, mitochondria and stem cells during ageing. Nature, 464,520-528.

Simon, N.M., Smoller, J.W., McNamara, K.L., Maser, R.S., Zalta, A.K., Pollack, M.H., Nierenberg, A.A., Fava, M., \& Wong KK. (2006). Telomere shortening and mood disorders: preliminary support for a chronic stress model of accelerated aging. Biological Psychiatry, 60, 432-435.

Teyssier, J.R., Ragot, S., Chauvet-Gélinier, J.C., Trojak, B., \& Bonin, B. (2011). Expression of oxidative stress-response genes is not activated in the prefrontal cortex of patients with depressive disorder. Psychiatry Research, 186, 244-247.

Wikgren, M., Maripuu, M., Karlsson, T., Nordfjäll, K., Bergdahl, J., Hultdin, J., Del-Favero, J., Roos, G., Nilsson, L.G., Adolfsson, R., \& Norrback, K.F. (2012). Short telomeres in depression and the general population are associated with a hypocortisolemic state. Biological Psychiatry, 71,294-300.

Wolkowitz, O.M., Mellon, S.H., Epel, E.S., Lin, J., Dhabhar, F.S., Su, Y., Reus, V.I., Rosser, R., Burke, H.M., Kupferman, E., Compagnone, M., Nelson, J.C., \& Blackburn, E.H. (2011). Leucocyte telomere length in major depression: correlations with chronicity, inflammation and oxidative stress preliminary findings. PloS ONE, 6, e17837.

Wolkowitz, O.M., Reus, V.I., \& Mellon, S.H. (2011). Of sound mind and body: depression, disease, and accelerated aging. Dialogues in Clinical Neurosciences, 13, 25-39.

Zhu, H., Belcher, M., \& van der Harst, P. (2011). Healthy aging and disease: role for telomere biology? Clinical Science, 120, 427-440. 